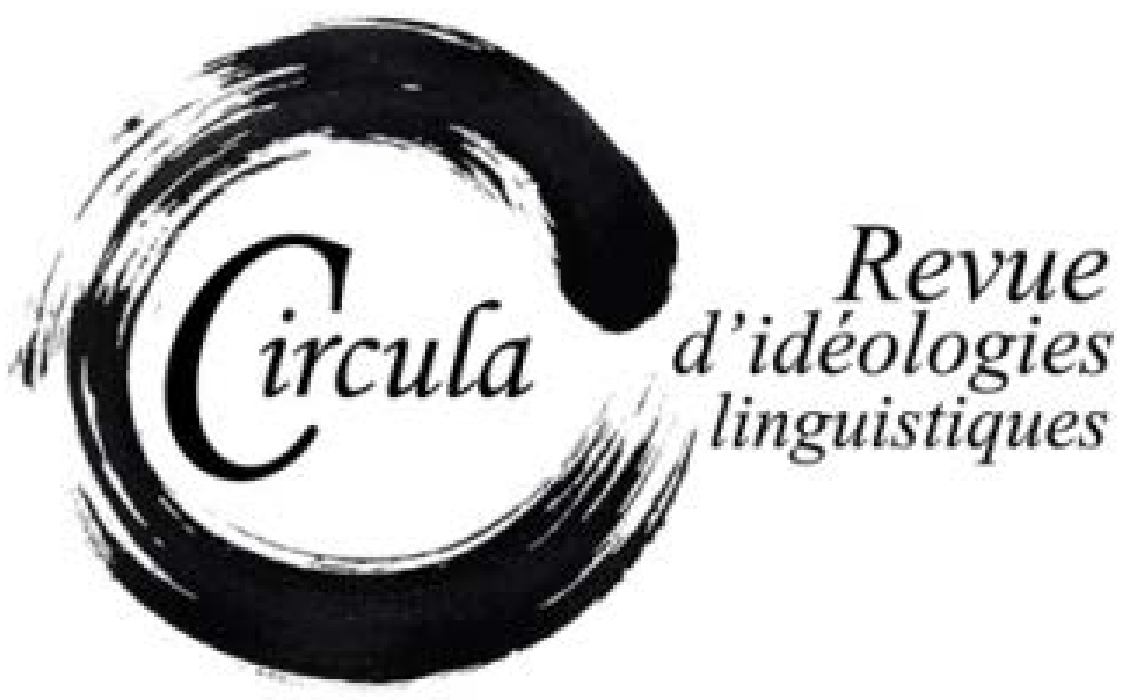

TITRE: UN'IDEOLOGIA LINGUISTICA SOMMERSA: LA QUESTIONE DELLA LINGUA (NON SOLO FILMICA) NEI PERIODICI CINEMATOGRAFICI ITALIANI TRA IL 1936 E IL 1945

Auteur(s): FABIO ROSSI, UnIVERSITÀ dI MESSINA

Revue: CirCula, NUMÉRO 5

PAgES: $83-106$

ISSN: 2369-6761

Directeurs: Wim Remysen, Sabine SCHWARZE et JuAn Antonio EnNiS

URI: HTTP://HDL.HANDLE.NET/11143/11229

DOI: HTTPS://DOI.ORG/10.17118/11143/11229 


\section{Un'ideologia linguistica sommersa: la questione della lingua (non solo filmica) nei periodici cinematografici italiani tra il 1936 e il 1945}

Fabio Rossi, Università di Messina

frossi @unime.it

Riassunto: Gli interventi di critici cinematografici tra il 1936 e il 1945 nelle principali riviste di cinema italiane, su argomenti di carattere linguistico, precedono di qualche anno la grande stagione del Neorealismo. II presente contributo, sulla base di 112 articoli raccolti in cinque periodici, mostra l'importanza e la precocità delle discussioni sull'italiano dello schermo. Si tratta di una porzione della questione della lingua e delle ideologie linguistiche finora trascurata dagli studi e, per questo, di grande interesse. I filmologi italiani hanno inaugurato argomenti poi destinati ad ampia fortuna, quali il ruolo e il funzionamento della parola rispetto all'immagine, il doppiaggio, il rapporto tra parlato, scritto e trasmesso, la necessità di una lingua per la comunicazione pubblica e media comprensibile a tutti, il rapporto tra italiano e dialetti. Nella loro veste di addetti ai lavori di un mezzo di massa, gli articolisti qui analizzati hanno compreso, prima dei linguisti, l'urgenza di reperire un codice quasi paradossale: realistico e comprensibile, naturale e universale.

Parole chiave: cinema; dialetto; doppiaggio; scritto/parlato/trasmesso; semiotica

Abstract: Film critics' articles on linguistic topics, written between 1936 and 1945 in the most important Italian film magazines, are very interesting because were produced a few years before the great season of Neorealism. This paper analyzes 112 articles collected in five magazines and shed light on the importance and earliness of the debate on the search for the Italian language fit to screen. This debate represents a part of the "Italian language affair" (Questione della lingua) and linguistic ideologies so far neglected and therefore very interesting. Italian film critics paved the way for new topics of large fortune, such as: the role and functioning of words compared to images; dubbing; the relationship between spoken, written and media language; the search for a public and everyday standard Italian, necessary for the media and understandable to everyone; the relationship between Italian and dialects. The columnists here analyzed, as involved in a mass medium (cinema), were able, before the linguists, to find a language almost paradoxical: realistic and understandable, natural and universal.

Keywords: cinema; dialect; dubbing; written/spoken/broadcasted discourse; semiotics 


\section{Introduzione: obiettivi della ricerca, ambito cronologico e de- scrizione del corpus}

La questione della lingua filmica occupa un posto non trascurabile nell'ambito della questione della lingua ${ }^{1}$. Quest'ultima, com'è noto, costituisce parte integrante della storia italiana e del sistema culturale italiano almeno da Dante a Pasolini (1991). Tale importanza di entrambe le questioni si deve, da un lato, al fatto che gli italiani, in tutta la loro storia, non hanno mai rinunciato a una spiccata metariflessione linguistico-culturale; dall'altro, al fatto che il nostro cinema si è sempre distinto per una vocazione, prima ancora che realistica, anch'essa metalinguistica e metaculturale, mediante «una vicinanza assoluta tra forme di rappresentazione e forme di vita» (De Gaetano, 2014: 28). Vocazione, questa, sicuramente superiore rispetto ad altre tradizioni filmografiche².

Scopo del presente contributo è quello di portare alla luce una porzione di riflessione linguistico-culturale italiana quasi del tutto sconosciuta, per mostrarne l'elevato interesse legato anche ai temi trattati, sotto descritti in dettaglio. Per conseguire tale obiettivo, abbiamo raccolto ed esaminato tutti gli articoli contenenti osservazioni linguistiche, pubblicati nelle principali riviste cinematografiche italiane degli anni trenta e quaranta del secolo scorso. Vediamo in dettaglio le testate scelte per la nostra indagine, lette per esteso in tutte le annate considerate, qui elencate in ordine cronologico di fondazione:

1. Oltre ai lavori di Sergio Raffaelli, tra i primi ad interessarsi della lingua filmica, riportati nella seconda sezione bibliografica a conclusione del presente articolo, ci si permette di rinviare anche ai titoli di Fabio Rossi, dedicati al linguaggio cinematografico e ai rapporti tra italiano filmico, italiano letterario e italiano comune (cf. soprattutto Rossi, 1999, 2006, 2015b, 2016).

2. Il cinema italiano è sempre stato «un cinema capace di percorrere quel sentiero stretto fra sentimento della vita e forme del mondo, avendo sentore e intuizione che nel primo (nel suo carattere liminare) si trovano sempre le condizioni per cambiare le seconde» (De Gaetano, 2014: 15). La forza innovatrice del cinema italiano, esplosa col Neorealismo, «risiede anche nella capacità di dirci qualcosa sul cinema in generale, di metterne in luce l'essenza» (De Gaetano, 2014: 17). Ma l'adesione al sentimento della vita e alle forme del mondo è un'«adesione scettica», raccontata quasi sempre in forma «autoriflessiva» (dal cinema critico e di poesia di Antonioni, per esempio) e autoparodica, dalla commedia all'italiana e dal cinema di genere (spaghetti-western ecc.) (De Gaetano, 2014: 23 ss.). 
- L'illustrazione italiana («rivista settimanale degli avvenimenti e personaggi contemporanei sopra la storia del giorno, la vita pubblica e sociale, scienze, belle arti, geografia e viaggi, teatri, musica, mode» [ecc.] dal 1875, Milano, Treves);

- Scenario («rivista mensile delle arti, della scena» dal 1932, Milano, Treves);

- Lo schermo («rassegna mensile della cinematografia» dal 1935, Roma, s.e.);

- Cinema («quindicinale di divulgazione cinematografica» dal 1936, Milano, Hoepli);

- Bianco e nero («quaderni mensili del Centro sperimentale di cinematografia» dal 1937, Roma, Laboremus).

Il totale degli articoli raccolti nelle succitate riviste, nel decennio 1936-1945, è di centododiciª Ciascuno di essi è stato scelto perché contiene almeno una notazione interessante dal punto di vista dell'ideologia linguistica (nell'accezione fornita in nota 6), vuoi perché è dedicato ai tecnicismi del cinema, vuoi perché tratta della lingua o dello stile dei dialoghi del film.

Violano le restrizioni del corpus quattro articoli: tre di Pirandello, del 1921 e del 1929 (scelti perché poi richiamati esplicitamente in articoli successivi delle nostre riviste) e uno di Luigi Comencini (1938), nel quindicinale Vita giovanile (scelto perché cruciale sul tema del parlato filmico)

Ci si chiederanno le ragioni della scelta di questo intervallo cronologico 1936-1945 e delle riviste selezionate. Nel 1936, come s'è visto, nasce il quindicinale Cinema, nel 1937 nasce il mensile Bianco e nero (vale a dire i due periodici cinematografici più autorevoli, quelli nei quali verranno messi a punto i prodromi del rinnovamento filmico italiano e anche della figura del critico cinematografico) e prendono vita, fin dai primi numeri, quelle istanze realistiche e metariflessive che condurranno all'affermazione del Neorealismo. Particolarmente importanti, in tal senso, sono gli articoli che caldeggiano l'adozione di una lingua filmica il più possibile vicina a quella parlata di tutti i giorni (Longanesi, 1936; Milano, 1938), come anche gli scritti che esaltano l'importanza del documentario (ancora Longanesi, 1936; De Feo, 1938; Vecchietti, 1938) e quelli che riconoscono al doppiaggio il merito di aver contribuito allo svecchiamento dei dialoghi filmici in direzione della velocità, della naturalezza e dell'espressività (Briareo, 1937; Franci, 1938c). Gli altri tre periodici sono stati scelti perché comunque altamente rappresentativi della critica cinematografica dell'epoca ${ }^{5}$. Interrompiamo la raccolta nel 1945 proprio perché, a Neorealismo consolidato, il dibattito culturale sui temi linguistici diventerà al contempo meno carsico (e dunque anche più noto agli studiosi) ma anche forse meno interessante,

3. Questo è il numero di articoli per anno: 1929: 1; 1936: 15; 1937: 11; 1938: 16; 1939: 11; 1940: 21; 1941: 19; 1942: 5; 1943 : 9; 1944: 2; 1945: 2.

4. «Periodico quindicinale di letteratura, arte, politica», fondato da Ernesto Treccani, Milano, Moneta, uscito tra il 1938 e il 1940. Dal n 16 (15 ottobre 1938) all’ultimo numero, il 9 dell'anno III (31 maggio 1940), cambiò il titolo in: «Corrente di vita giovanile».

5. Sull'importanza delle prime riviste specialistiche di cinema, cf. Brunetta (2001), vol. 2, cap. Il cammino della critica verso il neorealismo, p. 197-230. 
proprio perché il conseguimento del parlato filmico verrà dato sempre più per scontato, nelle sue peculiarità.

Quello considerato è pertanto un periodo cruciale non solo per la storia filmica mondiale (segnato, com'è noto, da fecondi contrasti tra ambizioni propagandistiche e smanie di rinnovamento, tra nostalgia del muto e film iperparlati, tra estimatori dello stile calligrafico e propugnatori del cinema verità, tra autarchici e filoamericani), ma anche per quella linguistica, che vedrà di lì a qualche decennio la nascita, o quanto meno il riconoscimento, di una nuova varietà del diasistema peninsulare: l'italiano dell'uso medio o neostandard.

Le considerazioni degli articolisti oggetto del nostro studio colpiscono, spesso, per la precocità e l'acutezza, tanto più notevoli in quanto provengono non tanto da linguisti e italianisti, bensi perlopiù da intellettuali cinefili. Le loro idee sulla lingua, dunque, possono configurarsi come ideologie linguistiche «laiche» e, come tali, sono di grande interesse per l'ambito di pertinenza della presente rivista6.

Vediamo ora la consistenza numerica degli articoli selezionati rispetto alle riviste del corpus:

Cinema: 52 articoli

L'illustrazione italiana: 27

Bianco e nero: 11

Scenario: 10

Lo schermo: 10

Corriere della sera: 1

Vita giovanile: 1

\section{I temi principali}

Gli interventi sul parlato filmico nelle riviste sopra elencate hanno un ruolo non marginale tra i temi trattati, temi che coniugano speculazioni teoriche (l'estetica del colore e del suono, lo statuto del cinema come arte, la funzione documentaristica rispetto a quella funzionale ecc.) a problemi di ordine pratico, come il rapporto tra cinema, fotografia, opera lirica, teatro e radio, la progettazione di sale dotate di adeguati requisiti acustici, la descrizione degli incipienti esperimenti televisivi, il rapporto tra film e fonti letterarie, i problemi giuridico-economici del diritto d'autore ecc.

6. Il tema dei non linguisti che prendono posizione su fatti di lingua è stato dibattuto in Rossi (2015a). Una sintesi sulle contrapposizioni ideologiche tra propugnatori di un italiano letterario ed uniforme e fautori del mimetismo localistico (soprattutto negli autori teatrali e in testi prossimi alla riproduzione del parlato) e anche proposte per la retrodatazione dell'italiano regionale, popolare e dell'uso medio possono ora essere lette in Trifone (2017). In quanto segue, si dà ad ideologia linguistica l'accezione ampia di insieme delle riflessioni sulla lingua non soltanto dal punto di vista delle strutture e dei fenomeni ma anche da quello dell'ambito d'uso, delle valutazioni estetiche o etiche e, in generale, «della natura del suo rapporto con il mondo», puntualmente discussa in Santulli (2015: 57 et passim). 
Che tipo di lingua utilizzare nei dialoghi dei film italiani e quale italiano scegliere per il doppiaggio sono i cardini attorno ai quali ruota la gran parte degli articoli. Ecco, in dettaglio, i 18 temi prevalenti degli articoli e il numero di articoli per tema:

- $\quad$ lingua del doppiaggio: 32

- $\quad$ svalutazione della parola a favore dell'immagine: 24

- cinema e letteratura: 11

- parlato comune e parlato filmico (nel quale rientrano varie osservazioni anche su italiano e dialetti e su recitazione artefatta/naturalistica): 10

- purismo: 7

- recitazione teatrale/cinematografica: 6

- a favore di un cinema come arte della realtà: 4

- lessico cinematografico: 3

- dialetto: 3

- $\quad$ semiotica: 2

- contro l'improvvisazione: 2

- la parola nel muto: 2

- a favore del sonoro: 1

- $\quad$ estetica del suono: 1

- lingua comica: 1

- cambiamento dei titoli originali in quelli della versione italiana del film: 1

- $\quad$ pronuncia degli attori: 1

- contro un cinema realistico: 1 
Naturalmente questa scansione tematica si sforza di ritagliare un solo tema prevalente per articolo. Volendo dare una visione più fluida del materiale raccolto, con le inevitabili intersezioni di temi in uno stesso articolo, emerge uno spettro tematico dedicato soprattutto al rapporto tra norma e uso nell'italiano della prima metà del Novecento; il ruolo del cinema nella diffusione e nell'unificazione linguistica (ben prima dell'avvento della televisione); il ruolo della parola nel film sonoro, spesso, ancora negli anni quaranta, ritenuto decisamente accessorio rispetto all'immagine; il contrastato rapporto tra italiano e dialetti; il rapporto tra italiano scritto, parlato (e "parlato-parlato») e trasmes$\mathrm{SO}^{7}$; i limiti dei soggetti e delle sceneggiature; il rapporto, anch'esso tutt'altro che pacifico, tra lingua del teatro, lingua della letteratura e lingua del cinema.

Il tema più ricco, variegato e stimolante è, come abbiamo visto, quello del doppiaggio, che va ben oltre la superficiale polemica tra favorevoli o contrari alla prassi della doppiatura (cf. AA.W, 1941a-g). Emergono infatti, da questi articoli (cf. almeno Patuelli, 1936; Gherardi, 1936a; Chiarini, 1936; Cortini Viviani, 1936; Vecchietti, 1936; Chiarini et Vecchietti, 1936; Antonioni, 1940a, b, 1941), preziose considerazioni di carattere lessicale, semiologico e traduttologico utili ancor oggi. Il film sonoro, e in particolar modo quello trasposto da una lingua all'altra, per la sua stessa natura sociosemiotica e multimodale, diventa il terreno ideale per dibattere sui problemi del parlato rispetto allo scritto, nelle sue numerose sottovarietà, e sulle aspettative del pubblico (frames, tipi di adattamento culturale ecc.). Per esempio, l'arguto articolo di Patuelli (1936) inaugura una polemica (che continuerà almeno fino a Chiarini et Vecchietti, 1936) su due modalità traduttive inconciliabili: quella source oriented, col vantaggio del rispetto filologico dell'originale ma con lo svantaggio di spiazzare il pubblico di arrivo che può trovarsi di fronte a frames culturali che non gli appartengono; e quella target oriented, con il vantaggio di essere più vicina alle attese e agli orizzonti culturali del grande pubblico, ma con lo svantaggio di forzare e di addomesticare spesso troppo il testo originale. Il cinema italiano doppiato (per evidenti motivi commerciali) ha subito optato (e lo fa tuttora) per la seconda modalità, differentemente dalla traduzione di testi letterari e scientifici, che di norma preferisce la primå.

Il doppiaggio, e più in generale la tecnica della postsincronizzazione, costituisce dunque, all'interno del nostro corpus, un vero e proprio banco di prova per verificare la resistenza, o viceversa la necessità di aggiornamento, dei consolidati strumenti d'analisi estetica e semiologica. Il film doppiato, infatti, in modo ancora più evidente di quello sonoro tout court, metteva a repentaglio più d'un principio dell'estetica crociana (unitarietà dell'opera d'arte), induceva a confrontarsi sulla natura dei mezzi di comunicazione di massa, a maggior ragione se multimediali (immagini, parole, musica, suoni, rumori), e sul rapporto tra valore estetico e valore commerciale di un'opera, stimolava la riflessione sulla natura delle diverse lingue, sui problemi del contatto linguistico e sul rapporto tra lingua e cultura di

7. I riferimenti ai concetti di «parlato-parlato» (Nencioni), «italiano dell'uso medio» (Sabatini), «parlato trasmesso» (Sabatini), cui si potrebbe aggiungere almeno l'italiano «neostandard» (Berruto), il «neoitaliano» (Spinazzola) e altri ancora, sono talmente noti, nell'ambito degli studi linguistici, da poter essere omessi in questa sede, con un unico richiamo riassuntivo a Rossi (2015b).

8. Sulle caratteristiche della lingua doppiata cf. almeno Pavesi (2005); Rossi (2006a: 265-344); Massara (2007); Perego-Taylor (2012). 
un popolo (a maggior ragione in un clima protezionistico e propagandistico come quello fascista), rinverdiva i problemi storici della traduttologia; insomma, stimolava la riflessione sul funzionamento stesso della comunicazione verbale e non verbale.

La polemica sui film doppiati culmina nel 1940-1941, con i noti articoli di Michelangelo Antonioni (1940a, b, 1941). L'interesse della questione, com'è facile comprendere dal tono di tutti questi articoli, va ben oltre la critica cinematografica, la natura del doppiaggio e le questioni linguistiche e traduttologiche ad essa connesse, investendo, in realtà, il modo di guardare alla produzione di massa («arte per masse», come la chiama Antonioni non senza un certo disprezzo, in Antonioni, 1941) da parte degli intellettuali italiani. La posizione di Antonioni è emblematica della difficoltà (direi dell'incapacità) dell'intellettuale formatosi sull'estetica crociana di accettare la natura composita dei mezzi di comunicazione e di mettersi dalla parte della ricezione e non soltanto da quella dell'elaborazione formale; il fatto che il film possa essere considerato anche come un prodotto di svago (o di utilità) fa vacillare l'impianto argomentativo costruito sulle arti nobili (pittura, letteratura ecc.), viste come totalmente svincolate da ogni aspetto pratico e di rapporto col pubblico, tra le quali si vorrebbe annoverare anche il cinema:

La questione del doppiato sta tutta qui: nella considerazione in cui si tiene il cinematografo. Il cinematografo è un divertimento puro e semplice, nel senso letterale del termine? Allora doppiamo. Ė un'arte, sia pure una cosiddetta "arte per masse"? Non doppiamo. I guai cominciano quando si afferma che è l'una e l'altra cosa insieme (Antonioni, 1941).

Non è casuale né ininfluente il fatto che in tutti questi articoli non si tocchi quasi mai il tema della postsincronizzazione dei film italiani (appena sfiorato in Antonioni, 1940a e in AA.W., 1941c), ma soltanto quella della versione doppiata di film da una lingua all'altra. Pressoché tutti i film italiani, almeno dalla fine degli anni trenta alla fine degli anni ottanta, ivi compresi quelli di Antonioni oltreché i capolavori del Neorealismo (con parziale eccezione per La terra trema), erano, com’è noto, «doppiati», vale a dire che la colonna sonora originariamente impressa sulla pellicola simultaneamente alle riprese del film era completamente sostituita da un'altra colonna sonora ricreata negli studi di doppiaggio in fase di postproduzione. Come poteva Antonioni biasimare il doppiaggio dei film stranieri, accettando (se non nella teoria, nella pratica) quello dei film italiani? A parte l'eventualità, poco probabile, di un radicale cambiamento di rotta (Antonioni gira i suoi primi film anni dopo la stesura dei suoi articoli contro il doppiaggio e potrebbe, dunque, effettivamente, aver cambiato idea nel frattempo), tale contraddizione è possibile perché la postsincronizzazione dei film italiani era avvertita come parte integrante della tecnica cinematografica e come unica garanzia di buona qualità acustica del film, tanto da essere concepita dal regista come un tutt'uno con la lavorazione del film stesso, a differenza del doppiaggio del film straniero, eseguito di norma senza tener conto della volontà del regista né tanto meno degli sceneggiatori originari. Ė chiaro che tale dicotomia rende giustizia della filologia (vale a dire della volontà degli autori del film), ma non del principio estetico, più volte invocato dallo stesso Antonioni, secondo il quale: 
Il processo formativo dell'opera d'arte è univoco, il doppiaggio lo sdoppia dando vita ad un'unità artificiale, cioè immaginaria, artisticamente nulla. Difatti la recitazione, mimica e linguaggio, nasce nell'attore da una sola e contemporanea ispirazione. Né si può trovare giustificazione nel fatto che il cinema è - almeno secondo alcune teorie - arte di collaborazione e che pertanto anche il doppiaggio può essere considerato uno dei molti elementi che servono a produrlo. Perché è chiaro che, anche ammettendo la collaborazione, questa dovrà tendere a priori verso l'unità, non a posteriori, cioè ad opera compiuta, come avviene nel caso di un film doppiato (Antonioni, 1940a: 328-329).

Alla base dell'avversione degli intellettuali per il doppiaggio dei film stranieri e anche dell'insensibilità per la postsincronizzazione dei film italiani sembrano esservi sia certa noncuranza per il gradimento del grande pubblico, sia la svalutazione della componente verbale rispetto a quella iconica: i dialoghi del film possono anche essere trascurati (non compresi, nel caso di film stranieri; svincolati dalla mimica degli attori, nel caso di film italiani postsincronizzati), dal momento che la specificità estetica del film sta nell'immagine più che nella parola. L'avvento del sonoro, che fu, com'è noto, a lungo osteggiato dagli intellettuali, rimette in discussione l'intero armamentario semiotico ed estetico allestito, sulla base del cinema muto, per interpretare il testo filmico. Le lunghe polemiche sul doppiaggio, dunque, vanno lette alla luce di tale complessa ristrutturazione dello statuto critico del film. Una ristrutturazione nella quale gli aspetti linguistici giocano un ruolo tutt'altro che marginale. È infatti proprio la parola al cinema, nel suo contrasto con l'immagine e nei suoi rapporti con la parola letteraria, quella teatrale e quella della comunicazione ordinaria, a rendere urgente il ripensamento dei soggetti verso un nuovo realismo. Urgenza tanto più avvertita sul territorio italiano, per via delle spiccate differenze tra la lingua scritta e quella parlata (o meglio quelle parlate). Ed è soprattutto la parola a traghettare il cinema da arte di élite, o quantomeno mezzo intellettuale, a mezzo di comunicazione di massa. La nascita di nuovi media non alfabetici (la radio prima, il film sonoro poi, la televisione infine) induce dunque a ripensare il rapporto tra norma e uso, tra varietà e stili comunicativi e ad aggiornare, di conseguenza, le teorie linguistiche. In breve, porta ad interrogarsi con nuovo fervore sul funzionamento stesso del linguaggio. Inevitabile ricordare le parole dell'ultimo quaderno di Antonio Gramsci (1935), il quale individuava nella questione della lingua un riflesso di nuove dinamiche sociali, di ricerca del consenso e di ristrutturazione del rapporto tra classi dirigenti e popolo:

Ogni volta che affiora, in un modo o nell'altro, la quistione della lingua, significa che si sta imponendo una serie di altri problemi: la formazione e l'allargamento della classe dirigente, la necessità di stabilire rapporti piú intimi e sicuri tra i gruppi dirigenti e la massa popolare-nazionale, cioè di riorganizzare l'egemonia culturale (Gramsci, 1996: 129).

Visto in quest'ottica, il doppiaggio, e il film sonoro in genere, si fa metafora del discorso sociale e la questione della lingua filmica acquista un rilievo di primaria importanza nella storia italiana della prima metà del Novecento. 


\section{La ricerca di una lingua per lo schermo}

Negli articoli raccolti nel decennio considerato, a interessarci non sono tanto i detrattori dell'errore, i novelli puristi barricati contro le impurità della lingua filmica, quanto i convinti assertori di una specificità del parlato filmico rispetto alla lingua letteraria e teatrale. Negli scritti di alcuni di loro (Paolo Milano, Ettore Allodoli, Raffaello Patuelli, Giacomo Debenedetti e molti altri elencati nella bibliografia conclusiva), ci sembra di poter retrodatare di almeno un quarantennio talune ipotesi, proposte e teorie (quali per l'appunto quelle sull'italiano dell'uso medio, sulle varietà intermedie tra scritto e parlato, sugli italiani regionali ecc.) che, nel campo della storia della lingua italiana accademicamente intesa, verranno formulate non prima degli anni settanta. Nulla di strano che siano proprio cinefili e cineasti (dai registi ai critici cinematografici, dagli sceneggiatori agli intellettuali semplici amatori di cinema) a porsi il problema della lingua. Molti degli articolisti da noi raccolti combinano all'attività giornalistica quella di sceneggiatore e regista (Gherardi, Franci, Debenedetti, Antonioni, De Santis, Comencini: non a caso quasi tutti poi coinvolti in film neorealistici o vicini al Neorealismo). La questione dunque, per loro, lungi dall'essere meramente accademica, era viva e pulsante: da una lingua agile e scorrevole derivava verosimilmente un maggior apprezzamento del pubblico e dunque un maggior rientro economico del film.

Nel titolo del presente articolo, abbiamo definito sommersa la questione della lingua filmica. Essa lo è a più livelli. In primo luogo, tutti questi interventi sono poco noti anche agli studiosi di cinema, del tutto ignoti ai linguisti. Da questo materiale emerge un'importante porzione della questione della lingua italiana del Novecento, ideologicamente trasversale (da un lato le posizioni puristiche e autarchiche, di regime, di Adolfo Franci e Ettore Allodoli, dall'altra quelle di Giacomo Debenedetti o Paolo Milano, che caldeggiano l'esempio dell'agile parlato americano a scapito del paludato italiano filmico) e continuamente in bilico tra questioni teoriche, propaganda e senso pratico di una lingua già lucidamente individuata come veicolo della comunicazione di massa.

Ma a rendere sommerso l'argomento qui affrontato, e conseguentemente l'ideologia che ne è alla base, è anche la stessa natura degli articoli. Essi sono sparpagliati nelle cinque riviste considerate, non sono raccolti in rubriche (con l'eccezione della rubrica di recensioni di Adolfo Franci, «Uomini donne e fantasmi», nella rivista L'illustrazione italiana, peraltro irregolare e di temi variegatissimi, non certo specificamente linguistica). Pertanto è stato necessario affrontare la lettura di tutte le annate per esteso, senza neppure confidare su autori riconoscibili. Con le illustri eccezioni di Allodoli, Menarini, Migliorini e pochissimi altri, infatti, non si tratta di esperti linguisti, bensì di critici cinematografici oggi perlopiù dimenticati ${ }^{9}$. Non è dunque stato possibile limitarsi ai titoli che esplicitamente

9. Gli articoli di Allodoli (1937, 1938), Migliorini (1941) e Menarini (1942) possono essere ora letti, quasi tutti in versione integrale, in Rossi (2016), insieme con Chiarini (1936), Chiarini-Vecchietti (1936), Cortini Viviani (1936), Gherardi (1936a), Leistner (1936), Longanesi (1936), Patuelli (1936), Vecchietti (1936), Briareo (1937), Debenedetti (1937), Mandelstamm (1937), Mauro (1937), Salvioni (1937), Comencini (1938), Franci (1938b, 1939), Milano (1938), Pac. (1938b), Pannunzio (1938), Sanminiatelli (1939), Antonioni (1940a, 1941), Rossi (1940), Ramperti (1942), De Franciscis (1943b), Felice (1943b), Paolella (1943), S.A. (1944), Guarnaccia (1945a, b). 
alludessero a temi linguistici. Se così fosse stato, per esempio, sarebbero rimasti incogniti articoli interessanti come quello di Michelangelo Antonioni (1940a), Vita impossibile del signor Clark Costa, o quello di Adolfo Franci (1937b), Un bandito e un paio di gemelli, o ancora quello di Carlo A. Felice (1943b), Un'altra retata, dal cui titolo nulla di linguistico sembrerebbe emergere. Un numero tutto sommato così elevato di articoli raccolti (centododici) si deve anche alla particolare sensibilità degli autori del primo ventennio del sonoro di concentrarsi non soltanto sulle peculiarità dell'immagine ma anche su quelle dei dialoghi, anche quando questi ultimi vengono svalutati rispetto alle immagini: per una sorta di preterizione, infatti, spesso è proprio per negarne l'importanza che si descrivono attentamente le caratteristiche del parlato filmico.

Il nostro materiale sommerso consente, tra l'altro, di rettificare non pochi luoghi comuni ancora circolanti sul cinema e sulla lingua d'epoca fascista, ben meno monolitici di quanto si creda.

A volte di lingua non si parla che per poche righe, ma con osservazioni acutissime, com'è il caso della semisconosciuta Emilia Salvioni. La scrittrice emiliana osserva che nel cinema italiano, a differenza di quello americano, non c'è alcuna attenzione alle ragioni sociali dei personaggi: tutto è finto, come finti sono i fondali dipinti, il cartonaggio, per l'appunto:

è molto meglio, non c'è dubbio, una scena umana, sincera che si svolge davanti all'orribile pittura del solito imbianchino, che un dialogo falso, goffo, teatrale, impossibile ad avverarsi nella realtà quotidiana, anche se viene rappresentato in un'atmosfera viva e reale. Questo disagio lo sentite in quasi tutta la nostra produzione: una ragazza in cinematografo, non è la stessa cosa che una ragazza nella vita ma si trasforma nell'ingenua del teatro ottocentesco o nella deplorevole "soubrette" dell'opera buffa. [...] in nessuna casa italiana, in nessun ceto, per nessuna ragione si danno casi di quel genere [cioè come quelli che si vedono nei film italiani], la gente si parla in quel tono. Uomini e donne in genere, si rivolgono l'uno l'altro col "voi". Ci son volute le case americane a scoprire che in Italia ci si dà più spesso del tu o del lei ed hanno imposto l'uso di questi due pronomi ai traduttori sicché nei doppiati troviamo il "lei" adoperati [sic] quasi con esagerazione (Salvioni, 1937: 32).

Articolo davvero prezioso (e anche in questo caso del tutto opaco nel titolo: Una piaga: il cartonaggio, che sembra trattare soltanto di scenografia), perché documenta, tra l'altro, la situazione degli allocutivi filmici subito prima del divieto fascista del Lei nel $1938^{10}$.

10. L'allocutivo di cortesia Lei fu vietato (a favore del Voi) il 14 febbraio 1938 con Disposizione del Partito nazionale fascista (cf. Raffaelli, 1993 e 2000). Il divieto comportò anche curiosi voltafaccia, come quello noto dell'Allodoli, che nell'articolo nel 1937 (Allodoli, 1937: 10-11) preferiva il Lei al Voi, così come già nella grammatica Trabalza-Allodoli (1934), mentre un anno dopo plaudiva alla sparizione del Lei come «affermazione di cameratismo e di comunanza di fede che stringe nelle espressioni di colloquio e di scrittura tanti milioni di italiani» (Ruffin et D’Agostino, 1997: 70-71). 
Tra i centododici articoli raccolti, alcuni hanno natura più letteraria che linguistica. Tuttavia essi hanno un ruolo fondamentale per chi intenda saggiare la temperie ideologico-linguistica del periodo, dal momento che mostrano come proprio dalla rivalutazione di certi autori (Goldoni e Verga: cf. Alicata et De Santis, 1941a, b; Ramperti, 1943), e anche su certe imprecise interpretazioni critiche (come l'improbabile immagine di un Verga dialettale), si giunga agli albori del Neorealismo e dunque al rinnovamento stilistico, ma in primo luogo linguistico, del cinema italiano verso la fine del secondo conflitto mondiale.

\section{La prefigurazione dell'italiano dell'uso medio come anticipazio- ne del Neorealismo}

Per una lettura completa degli articoli più interessanti del corpus, come già detto, si rimanda a Rossi (2016). Cionondimeno, si riportano di seguito alcune citazioni particolarmente significative, a partire da un precoce e acuto articolo di Paolo Milano. Col nuovo mezzo del sonoro si ripresenta in tutta la sua urgenza pratica lo stesso problema già incontrato da Manzoni e Verga, non a caso citati nell'articolo: la ricerca di una lingua viva e parlata da contrapporre allo stantio italiano letterario:

Che linguaggio sceglierà il Cinema, fra i molti che ogni lingua possiede? Il più semplice, il più documentario, il più legato all'esistenza spicciola e quotidiana. Qualunque altro linguaggio più sostenuto, letterario o (come si suol dire) aulico, rischierebbe d'assumere un valore artistico proprio, a tutto scapito della visione filmica, in ibrido e sterile connubio. [...]

Così stando le cose, gli americani sono a cavallo. Quando si loda il dialogo dei loro film, si pensa di solito al frizzante delle battute, alla mirabile (sebbene un po' frigida) loro tecnica della ripetizione, dell'analogia, del richiamo. Ma assai più notevole, e meglio efficace, è la lingua che i personaggi parlano: quel gergo disossato e breve che sembra fatto di ammiccamenti e di urti più che di parole, quell'inglese d'oltresponda diventato irrispettoso e pregnante. Ė la lingua cinematografica per eccellenza, sia detto senza complimento: cioè la lingua più lontana dalla poesia. [...]

Ora, sarebbe tempo che anche il dialoghista cinematografico si associasse con lena e buon diritto a un'opera che si prosegue da più di un secolo, alla quale hanno contribuito e Manzoni e Verga e Pirandello, e a cui lavorano più o meno inconsapevolmente giornalisti e padri di famiglia e uomini della strada: la creazione di una lingua italiana di tutti i giorni.

A che punto sta quest'opera collettiva? Un pezzo avanti, mi sembra. Intanto, i rapporti sempre più fitti fra regione e regione hanno creato una specie di fondo linguistico comune, a mezza strada fra lingua e dialetto. [...] 
Perché questo è il problema del linguaggio cinematografico: il personaggio dello schermo deve parlare come quello che lo spettatore incontra ogni giorno a un angolo di strada, al caffè, in ufficio, in un salotto. Propongo una multa per il primo sceneggiatore che ancora una volta metterà in bocca a un personaggio di film una frase come «Ho detto loro...». Vergogna! Sullo schermo si dice, anche al plurale e in barba alla Crusca, «gli ho detto», e si resta in ottima compagnia, visto che Manzoni l'ha scritto tante volte (Milano, 1938).

L'esempio (gli per loro) addotto dal Milano a conferma della necessità «di una lingua italiana di tutti i giorni» (Milano, 1938: 11, in corsivo nell'originale) costituisce una vexata quaestio nella questione della lingua (cf. Palermo, 2006). Oltre al passaggio manzoniano, dalla Ventisettana alla Quarantana, di pochi loro trasformati in $\mathrm{g} / i^{11}$, spiccano le parole del Fornaciari:

Il popolo toscano nel parlar familiare usa non di rado gli per le (a lei), e quasi sempre gli per a loro, modi condannati dai grammatici e rari nei buoni scrittori, specialmente degli ultimi tre secoli. Quanto al primo gli, stimiamo che se ne debba vietar l'uso assolutamente, si perché le persone civili adoprano, parlando, anche le, e perché, oltre a togliere ogni equivoco, le è breve e spedito quanto gli, ed è vera forma congiuntiva [clitica] né più né meno. Il secondo gli ha a proprio favore una ragione assai buona; cioè che loro (nel senso di a loro) con cui i libri lo sostituiscono, non è congiuntiva, ma, per quanto si accorci in lor premesso al verbo, resta sempre una forma assoluta [piena e non clitica] e pesante, ed in certi casi insopportabile, come quando si trova vicino ad un altro loro (Fornaciari, 1881: 53).

Ancor più nettamente favorevole a gli era stato il Tommaseo di Fede e bellezza (1840/1852): «Ma queste, lo veggo, son precauzioni simili a quelle di certi vostri politicanti, buone finch'altre ragioni più intime gli dieno (dovrei dire dien loro, ma mi par tanto letterato quel loro!) gli dieno virtù» (BIZ).

Meno tollerante il Tommaseo-Bellini, s.v. gli (pronome), § 3: «Talora però si trova posto per terzo caso del genere masculino, nel num. del più, come usa il volgo, ma è creduto modo di dire poco regolato» (BIZ).

Bisognerà tuttavia, prima dei nostri giornalisti filmici, aspettare qualche grammatico anticonvenzionale d'inizio secolo, per una prima convinta affermazione di gli a scapito di loro, ovvero del parlato rispetto allo scritto, dell'uso vivo rispetto a quello letterario e imposto dai grammatici:

le opere son giovani e la grammatica è vecchia, e tra i giovani e i vecchi, si sa, non si può andar sempre d'accordo. Nel meglio della lezione, fermandosi, per esempio, su un passo come questo: «E sui rami erano posati uccelli di tutti i colori, che non gli mancava che il fischiare», il raccoglitore in una nota è costretto ad ammonire: «che non gli: si dovrebbe dire: a cui o ai quali non mancava. O almeno almeno: non mancava loro. Ma... non si dice!». Non si dice! - E se non si dice, - pensan gli alunni, - come e perché la grammatica pretenda che si dica? [...]

11. Serianni (1989: 194-195) osserva giustamente come «sopravvalutare l'importanza dei pochi gli accolti dal Manzoni nella quarantana significa compiere un errore di prospettiva». 
Or io, modestamente, ho cercato di levar questa contraddizione. Uno degli scopi del presente lavoro è stato appunto di spogliar questo insegnamento di tutto quell'orrore ridicolo per le forme schiette e spigliate della lingua viva, e persuadere gli alunni che la grammatica può anche non essere la befana (Cesare De Titta, Grammatica italiana della lingua viva per uso nelle scuole ginnasiali, tecniche e complementari, 1901, cit. in Demartini, 2014: 68).

Per tornare ai nostri articolisti cinematografici, similmente a Paolo Milano, nello stesso 1938, Luigi Comencini così concludeva una sua rassegna sul festival di Venezia, di fatto anticipando le istanze neorealistiche:

È necessario ritrovare la vita italiana, e non nei libri e nelle antologie [...]. Gli italiani non parlano l'italiano; generalmente parlano un dialetto; e ad ogni modo sempre una lingua che è parlata e non scritta [...]. II dialoghista [...] deve essere una persona geniale, che sappia parlare come gli operai, come gli impiegati e pensare come un grande scrittore, che non abbia paura delle parole [...]. Il più bel film italiano si svolge ancora nelle piazze e nelle vie d'Italia dove la gente parla a crocchi [...]; nei campi dove lavora, e nei caffè [...], dove si riunisce la sera (Comencini, 1938).

Non è difficile prefigurare, nelle parole di Comencini (1938), Milano (1938) e altri, quelle istanze che, di lì a qualche anno, sarebbero state formulate da Cesare Zavattini come poetica del «pedinamento della realtà»: «il tempo è maturo per buttare via i copioni e per pedinare gli uomini con la macchina da presa» ${ }^{12}$. Tra le prime formulazioni di tale poetica, va ricordato almeno Longanesi (1936), sulla necessità di «sorprendere la realtà», anche linguistica, del nuovo cinema italiano:

Benché io non sia un tecnico del film, ed abbia a noia ogni dilettante e, per di più, ogni cinedilettante ed ogni pellicola fatta in casa, mi sento a volte 'portato verso il documentario' e penso ora a questo, ora a quel soggetto perché stando seduto ore e ore al caffè come faccio, non mi manca l'occasione di vedere passare sotto i miei occhi straordinarie scene e magnifici personaggi anonimi.

Giorni fa, a un caffè di piazza del Popolo, avevo alla mia destra, seduti a un tavolo, due uomini e una ragazza. Parlavano grasso, come s'usa dire, bevendo a tratti qualche boccata di birra, e ridendo a gola aperta. La donna mangiò un cannellone alla crema e si macchiò l'abito. Tutti e tre risero. Uno dei due uomini che aveva le mani grassocce come quelle di un putto, con certe unghie corte, schiacciate e lucide che sembravano disegnate da Grosz, disse: «Sono stato dalla manicure» e mostrò le sue unghie color rosa.

«Quando si guadagna non si bada a spese» esclamò l'amico.

«Si fa quello che si può» rispose il primo. E giù risate grasse e sonore. Poi la conversazione cade sulle donne. Quello con le unghie rosa dice: «La donna deve essere femmina, deve avere dello slancio». L'altro, l'amico, piegando il capo verso la ragazza e strizzandole l'occhio chiede:

12. Dichiarazione del 1951, in Zavattini (1979: 83); cf. peraltro l'intero volume, e in partic. le p. 31-40, per analoghe considerazioni zavattiniane già alla fine degli anni trenta. 
«Lei ha slancio?

«Secondo con chi» risponde la ragazza, e giù risate.

Ebbene, se io fossi un operatore girerei per strada con la macchina da presa e coglierei scene di questo genere, stenografando i dialoghi su un taccuino.

Per farne che? Per allestire un documentario sulla vita degli anonimi. 'La vita degli anonimi'. Ecco un titolo alla Fabre. E come Fabre osservava gli insetti, osserverei questi personaggi della strada. I miei tre visini, la ragazza vestita di viola e i due commercianti, avevano una vivacità di gesti, un linguaggio, una certa maniera di prendere la vita che nessun film è riuscito mai a mostrarci. La verità che li animava non si può ricostruire, non si può insegnare ad un attore: è una verità, come dire?, d'istinto, fatta costume dall'abitudine e dalla pratica; essi raggiungono uno stile brutale, di infimo ordine, greggio, ma sempre uno stile che rivela l'assoluta impossibilità di critica e di controllo. lo avrei seguito questi tre personaggi quando, lasciato il caffè, salirono in carrozza e scomparvero verso Villa Borghese. Ma non avrei cercato di scoprire di più di quel che tutti potevano vedere; non avrei intrapreso la ricerca dei loro caratteri né tessuto la trama di una immaginaria vicenda; quel che con la macchina avrei còlto di sfuggita, mi sarebbe bastato per aprire il mio documentario. Poi, non avrei incontrato eccessive difficoltà per scoprire cento altri aspetti della vita quotidiana di questi anonimi personaggi che vivono senza sospetto in tutte le vie e in tutti i caffè. E a poco a poco avrei cercato così di mostrare, attraverso qualche centinaio di metri di pellicola, la felicità fisica, la felicità senza scrupoli, la felicità insomma di chi si affida al moto dell'esistenza e si lascia trasportare senza eccessive esigenze.

Ma è proprio necessario, a questo mondo, mostrare la verità? (Longanesi, 1936: 258-260).

\section{Verbale e non verbale}

In un manipolo di articoli, non sono tanto le considerazioni linguistiche nel senso tradizionale del termine, ad essere al centro dell'attenzione dei nostri autori, bensì attente ricognizioni sul funzionamento del linguaggio verbale, messo a confronto con quello filmico e con altri linguaggi non verbali. È il caso di Roberto Paolella:

lo scrittore impiegando una fraseologia tesa e cerebrale, usa esclusivamente la subordinazione propria degli schemi sintattici, in cui le leggi profonde della gesticolazione spontanea sono invertite e sconvolte.

Ove lo scrittore costruisce una frase come questa: «l'uomo che vedete là basso sulla spiaggia, è quello che ho incontrato alla stazione», il compositore orale dice. «Vedete quell'uomo là basso? Egli è seduto sulla spiaggia. Ebbene, io l'ho incontrato alla stazione» (Vendryes). Qui la maniera di esprimersi è ancora la recitazione gestuale dell'azione e cioè una pura sequenza che l'attuale linguaggio cinematografico traduce ancora oggi così: 
1. lungo fuoco della spiaggia coll'uomo seduto;

2. mezzo primo piano dell'uomo seduto;

3. sovrimpressione per reviviscenza: incontro dell'uomo alla stazione.

La lingua parassitaria scritta non è dunque [...] in equilibrio con l'ideazione naturale. Essa esige una tensione cerebrale opprimente ed esaurisce rapidamente il pensiero. L'arte cinematografica, ripristinando le leggi profonde della gesticolazione spontanea, costituisce un ritorno all'indietro che è insieme un progresso e una giusta reazione della natura (Paolella, 1943: 141).

L'articolo appena citato si mostra, da un lato, particolarmente precoce nel presentare alcune tendenze aggiornate della linguistica (nella fattispecie, le osservazioni di psicolinguistica dell'antropologo francese Marcel Jousse, uno dei primi studiosi del codice gestuale), come per esempio la pragmatica e il concetto di atto linguistico: «nello stile orale, recitare delle parole significa compiere ciò che esse dicono. (Es. fare una preghiera)» (Paolella, 1943: 140), oltreché le acute osservazioni sulla differenza tra sintassi additiva e ancorata allo spazio-tempo (deissi) nel parlato, rispetto alle manipolazioni ipotattiche dello scritto; dall'altro, però, risente del solito pregiudizio estetico che relega la parola del film ad aspetti marginali, quando non deteriori: «col sonoro sopravviene la catastrofe, perché il nuovo mezzo non risponde a niente. Non è mai il personaggio che parla, ma solo il quadrato luminoso ove esso è proiettato» (Paolella, 1943: 142). Resta notevole che, a far arrivare alcune aggiornate teorie linguistiche in Italia, sia una rivista di cinema, a conferma, se ancora ce ne fosse bisogno, della notevole apertura culturale e dell'ottimo livello del dibattito critico nelle riviste cinematografiche degli anni trenta-quaranta; e a conferma, ancora una volta, dell'utilità di questo materiale per una storia delle ideologie linguistiche.

\section{Considerazioni conclusive}

Siamo partiti dal presupposto che gli articoli scritti negli anni trenta e quaranta del Novecento, nei principali periodici cinematografici italiani, avessero un interesse non soltanto limitato alla critica e alla filmologia, ma fossero utili anche ai linguisti, tanto più se interessati alle ideologie linguistiche, per ricostruire una porzione cruciale, benché pochissimo nota, della questione della lingua italiana contemporanea. I centododici articoli raccolti e commentati in questa sede confermano pienamente l'interesse e la precocità degli argomenti trattati, che spaziano dalla grammatica alla pragmatica, dalle osservazioni semiotiche sui rapporti tra parole e immagini al funzionamento del doppiaggio, dal rapporto tra parlato, scritto e trasmesso al rapporto tra italiano e dialetti, dalle osservazioni sociolinguistiche sulla comunicazione pubblica e la lingua dei media alla precoce individuazione di un italiano dell'uso medio. 
I nostri articoli esibiscono inoltre una folta messe di fenomeni linguistici a quattro diversi livelli:

1) la lingua del cinema, ovvero il lessico specifico delle tecniche di ripresa e proiezione: in questa direzione vanno gli articoli di Migliorini e di Menarini (cf. Migliorini, 1941 e Menarini, 1942) su tecnicismi quali film o filme, doppiaggio o doppiato, mischiatura in luogo di mixage ecc. e le lunghe liste di prescrizione fascista dei forestierismi. Grazie ai nostri articoli è possibile anche reperire termini spesso sfuggiti alle maglie dei dizionari, o comunque attestati troppo tardivamente, com'è il caso dei tecnicismi sincroneta (1936), cartonaggio (1937) e molti altri (doppiatore 1936, sovrimpresso 1936, fonogenia 1936, disincronizzazione 1937, prestavoce 1937, ciak 1938, missare 1941, e ancora: arredatore, bobinare, ciacchista, cinecronaca, fonogeno, trucco, tutti del 1942, e altri ancora, per i quali si rimanda a Rossi, 2016: 19-20).

2) La lingua nel cinema: ovvero come debbono parlare gli attori sul grande schermo e quali sono le peculiarità del parlato trasmesso rispetto al parlato-parlato e allo scritto teatrale e letterario.

3) La lingua dal cinema: qual è la ricaduta delle battute cinematografiche sulla lingua di tutti i gior$\mathrm{ni}^{13}$.

4) La lingua sul cinema: matura, nel decennio considerato, l'italiano della critica cinematografica, esemplificato proprio dal nostro corpus. Spicca, al riguardo, una tendenza all'analisi e alla speculazione, anche di natura linguistica e semiologica, oggi pressoché scomparsa nella nostra critica cinematografica. Non è infrequente imbattersi, nelle recensioni dell'epoca, in sezioni interamente dedicate ai dialoghi (magari soltanto per screditarli o per metterne in luce l'inutile verbosità rispetto all'immagine) e al doppiaggio. Anche gli articoli di taglio più divulgativo, pure quando indulgono alla cronaca e al pettegolezzo, raramente sono privi di argomentazioni argute, di riferimenti culturali, di informate notazioni tecniche: «La disamina tecnica diventa spesso riflessione estetica quando non si apra a spunti mediologici di singolare lungimiranza, del tutto inconsueti nel panorama della cultura italiana dell'epoca» (Caldiron, 2002:11), per non dire di quella attuale; né abbassano mai la guardia stilistica sotto una certa soglia.

Il nostro corpus mostra con grande evidenza un tratto assolutamente caratteristico dell'intera nostra storia linguistica, quello della quête: gli italiani, da Dante in poi, sono sempre andati alla ricerca della giusta lingua ${ }^{14}$ : dapprima il volgare illustre adatto alla canzone; poi, tra Sei e Ottocento, una lingua adatta alla prosa, d’arte e scientifica; in seguito, tra Otto e Novecento, una lingua per il teatro,

13. Mentre i primi tre livelli, sebbene qui un po' forzati e ampliati, erano già stati individuati da Raffaelli (1992: 48, ma la prima versione dell'articolo è del 1983, con debiti precedenti), il successivo quarto livello viene messo qui a punto per la prima volta.

14. «In cerca della lingua» è il titolo del paragrafo 2.3 di Raffaelli (2015). 
tragico e comico ${ }^{15}$; e infine, a partire da Manzoni, un italiano dell'uso, scritto e parlato, comune a tutti gli italiani. Tutto l'ampio dibattito presente nelle nostre riviste di cinema non è dunque che l'atto conclusivo di un percorso, secolare e coerente, di ricerca linguistica, e dunque di ideologia linguistica. E non meravigli che siano stati proprio i cineasti (parallelamente a quello che i grammatici stavano facendo già da qualche decennio: cf. Demartini 2014) a cimentarsi su argomenti quali il ruolo e il funzionamento della parola, il rapporto tra parlato e scritto e l'intelligibilità di tutti gli italiani. Nessuno, infatti, meglio degli addetti ai lavori di un mezzo di massa può comprendere l'urgenza di reperire un codice al contempo realistico, medio, colloquiale e comprensibile a tutti, italiano ma non letterario, recitato ma non teatrale, parlato, regionale e talora popolare ma non integralmente dialettale, facilmente intelligibile ma non innaturale, naturale ma universale.

15. Non a caso, quanti, nell'Ottocento, lamentavano l'inesistenza di una letteratura popolare in Italia, connettendola all'assenza di un parlato sovraregionale unitario, criticavano (forse un po' troppo avventatamente, dimenticando Goldoni, i goldoniani e soprattutto l'opera buffa) anche la sostanziale latitanza di un teatro comico italiano (rispetto a quelli francese e dialettale): «Se tu leggi qualche commedia moderna italiana, tu vedrai che il più comico è l'impaccio dell'autore a trovare le sue parole» (Bonghi, 1856/1884: 243; su temi analoghi cf. anche Trifone, 2017). La ricerca di un parlato filmico credibile e funzionale al nuovo mezzo di comunicazione sembra dunque il coerente sviluppo di quelle critiche, come mostrano anche i numerosi punti di tangenza tra osservazioni filmiche e teatrali nei nostri articoli (per la lingua teatrale italiana cf. da ultimi Giovanardi et Trifone, 2015). 


\section{Bibliografia}

\section{Fonti primarie}

AA.W. (1941a), «Pro o contro? Inchiesta sul doppiaggio», Cinema, vol. 6, n’ 109, p. 10-11.

AA.W. (1941b), «Pro o contro? Inchiesta sul doppiato», Cinema, vol. 6, n 110, p. 48-49.

AA.V. (1941c), «Pro o contro? Inchiesta sul doppiato», Cinema, vol. 6, n 111, p. 85-86.

AA.WV. (1941d), «Pro o contro», Cinema, vol. 6, n 112, p. 120-121.

AA.VV. (1941e), «Pro o contro? Inchiesta sul doppiato», Cinema, vol. 6, n 113, p. 154-156.

AA.VV. (1941f), «Pro o contro? Inchiesta sul doppiato», Cinema, vol. 6, n’114, p. 190-192.

AA.V. (1941g), «Pro o contro? Inchiesta sul doppiato», Cinema, vol. 6, n’ 115, p. 226-228.

AA.W. (1941h), «Filologia del cinema», Bianco e nero», vol. 5, n’ 4, p. 93-99.

Alicata, Mario e Giuseppe De Santis (1941a), «Verità e poesia. Verga e il cinema italiano», Cinema, vol. $6, n^{\circ} 127$, p. 216-217.

Alicata, Mario e Giuseppe De Santis (1941b), «Ancora di Verga e del cinema italiano», Cinema, vol. 6, nं130, p. 314-315.

Allodoli, Ettore (1937), «Cinema e lingua italiana», Bianco e nero, vol. 1, n 4, p. 3-11.

Allodoli, Ettore (1938), «Cinema e lingua italiana», Bianco e nero, vol. 1, n’ 4, p. 43-47.

Altichieri, Gilberto (1938), «La lingua e il “parlato”», Cinema, vol. 3, n’ 40, p. 116.

Alvaro, Corrado (1936), «Grammatica del film», Scenario, vol. 5, n’ 4, p. 159-162.

Antonioni, Michelangelo (1940a), «Vita impossibile del signor Clark Costa», Cinema, vol. 5, n 105, p. 328-330.

Antonioni, Michelangelo (1940b), «Ultime note sul doppiaggio», Cinema, vol. 5, n 107, p. 399.

Antonioni, Michelangelo (1941), «Conclusioni sul doppiato», Cinema, vol. 6, n 116, p. 261.

Antonioni, Michelangelo e Gianni Puccini (1940), «Due lustri di sonoro», Cinema, vol. 5, n’ 108, p. 437440.

Arnheim, Rudolf (1937), «Sonoro o muto? "Ma che cosa è questo cinema?”, Cinema, vol. 2, n³3, p. 306.

Arnheim, Rudolf (1938), «Nuovo Laocoonte», Bianco e nero, vol. 2, nº, p. 3-33.

Balàzs, Bela [trad. Umberto Barbaro] (1940), «Lo spirito del film», Bianco e nero, vol. 4, n’ 2, p. 3-66. 
Bargellini, Piero (1943), «Le parole dipinte», Cinema, vol. 8, n 158, p. $42-43$.

Briareo, Gustavo [Giacomo Debenedetti] (1937), «ll doppiaggio in Italia», Cinema, vol. 2, n² 29, p. 154156.

Calcagno, Diego (1940), «ll problema delle voci», Cinema, vol. 5, n. 104, p. 293.

Callegari, Gian Paolo (1942), «Cinema e romanzo», L'illustrazione italiana, vol. 69, n³5, p. 238.

Cambi, Enzo (1939), «Qualità e quantità dei suoni», Cinema, vol. 4, n’ 83, p. 353-354.

Cappelletti, Franco (1939), «Voci e immagini», Cinema, vol. 4, n 74, p. 60-61.

Chaplin, Charles (1940), «Chaplin contro il parlato», Cinema, vol. 5, n’ 108, p. 448.

Chiarini, Luigi (1936), «Intraducibilità del film», Lo schermo, vol. 2, n 8, p. 30-32.

Chiarini, Luigi (1940), «La musica nell’unità del film», Cinema, vol. 5, n 108, p. 450-451.

Chiarini, Luigi e Giorgio Vecchietti [sotto lo pseudonimo di L’uomo ombra] (1936), «Pro e contro il doppiaggio. Mangiatori di minestrone», Lo schermo, vol. 2, n’11, p. 23-24.

Comencini, Luigi (1938), «La mostra cinematografica di Venezia», Vita giovanile, vol. 1, n’ 15, p. 6.

Corsi, Mario (1936), «Fregoli pioniere del muto e precursore del sonoro», Cinema, vol. 1, n¹1, p. 416417.

Cortini Viviani, Maria (1936), «l segreti del doppiaggio», Cinema, vol. 1, n 6, p. 232-233.

Dabini, Attilio (1940), «Dal pianino al grammofono», Cinema, vol. 5, n’ 108, p. 448.

D’Amico, Silvio (1939), «Pronunciare l'italiano», Scenario, vol. 8, n’ 9, p. 424-425.

Debenedetti, Giacomo (1937), «Primo punto: la sceneggiatura», Cinema, vol. 2, n³4, p. 335-337.

De Feo, Luciano (1938), «Documento di vita nei programmi», Cinema, vol. 3, n 39, p. 77-78.

De Franciscis, Umberto (1939), «Le parole», Cinema, vol. 4, n 74, p. 47.

De Franciscis, Umberto (1940), «Storia del dialogo», Cinema, vol. 5, n’ 108, p. 443-444.

De Franciscis, Umberto (1943a), «l personaggi di Donna Matilde», L'illustrazione italiana, vol. 70, n 15, p. 341-342.

De Franciscis, Umberto (1943b), «Suggeritori senza buca», Scenario, vol. 12, n¹1, p. 359-361.

De Santis, Giuseppe (1940), «L’ispirazione sensibile», Cinema, vol. 5, n’ 108, p. 453.

Ėizenštejn, Sergej M., Vsevolod I. Pudovkin e Grigorij V. Alexandrov [traduzione di Umberto Barbaro] (1940), «Un manifesto del 1928» [1928], Cinema, vol. 5, n¹08, p. 445.

Felice, Carlo A. (1943a), «La maniera dei più», L'illustrazione italiana, vol. 70, n’ 10, p. 234. 
Felice, Carlo A. (1943b), «Un’altra retata», L'illustrazione italiana, vol. 70, n²7, p. 573.

Felice, Carlo A. (1943c), «Novità N.N.», L'illustrazione italiana, vol. 70, n’32, p. 662.

Franci, Adolfo (1937a), «Discorsi e film di stagione», L'illustrazione italiana, vol. 64, n³2, p. 913.

Franci, Adolfo (1937b), «Un bandito e un paio di gemelli», L'illustrazione italiana, vol. 64, n’ 48, p. 1477.

Franci, Adolfo (1938a), «Un po’ di bilancio prima della cronaca», L'illustrazione italiana, vol. 65, n 5, p. 151.

Franci, Adolfo (1938b), «Del film italiano all'estero e del doppiato», L'illustrazione italiana, vol. 65, n'18, p. 643.

Franci, Adolfo (1938c), «Del film Olimpia e di altre cose», L'illustrazione italiana, vol. 65, n’26, p. 1077.

Franci, Adolfo (1939), «Parentesi filologica», L'illustrazione italiana, vol. 66, n’ 30, p. 153.

Franci, Adolfo (1940a), «Platone e Daudet sullo schermo», L'illustrazione italiana, vol. 67, n³, p. 88.

Franci, Adolfo (1940b), «Tre scrittori classici al soccorso del cinematografo», L'illustrazione italiana, vol. 67, n5, p. 147.

Franci, Adolfo (1940c), «Bizze e capricci della bella Viviane», L'illustrazione italiana, vol. 67, n’ 10, p. 307.

Franci, Adolfo (1941a), «Parole al vento e voci nella tempesta», L'illustrazione italiana, vol. 68, n 12 , p. 423.

Franci, Adolfo (1941b), «Discrete domande a Baldini», L'illustrazione italiana, vol. 68, n’26, p. 1023.

Franci, Adolfo (1941c), «Omaggio a Ucicky», L’illustrazione italiana, vol. 68, n² 27, p. 24.

Franci, Adolfo (1941d), «Del soggetto cinematografico e di altre cose», L'illustrazione italiana, vol. 68, n 35, p. 291.

Franci, Adolfo (1941e), «Cinema e letteratura», L'illustrazione italiana, vol. 68, n50, p. 731.

Gherardi, Gherardo (1936a), «Voci e figure di personaggi impossibili», L'illustrazione italiana, vol. 63, nं31, p. 204.

Gherardi, Gherardo (1936b), «Dialogo da scena e parole da schermo», Lo schermo, vol. 2, n 9, p. 37.

Gherardi, Gherardo (1936c), «Parlando del “parlato”», Cinema, vol. 1, n 8, p. 311.

Gherardi, Gherardo (1937), «Teoria del dialogo cinematografico», Bianco e nero, vol. 1, n 12, p. 3-9.

Gherardi, Gherardo (1939), «Prassi del dialogo cinematografico», Bianco e nero, vol. 3, n 5, p. 60-63.

Giani, Renato (1940), «La parola drammatica», Cinema, vol. 5, n¹08, p. 454.

Griffith, Richard (1940), «Sonoro e standard», Cinema, vol. 5, n 108, p. 442. 
Guarnaccia, Vincenzo (1945a), «Primo incontro con Charlot», L'illustrazione italiana, nuova serie, $n^{\circ} 8$, p. 137.

Guarnaccia, Vincenzo (1945b), «Limiti di Macario - Edison giovane - tirannia del doppiaggio», L'illustrazione italiana, nuova serie, $n^{\circ}$ 9, p. 157.

Hugon, Paul D. (1939), «Sintassi dello schermo», Cinema, vol. 4, n 78, p. 202-203.

Kaft, Bino (1940), «Lettere agli autori: il problema delle voci», Cinema, vol. 5, n’ 106, p. 367.

Leistner, Erick (1936), «Come si recita nel fonofilm», Cinema, vol. 1, n 7, p. 270-271.

Longanesi, Leo (1936), «Sorprendere la realtà», Cinema, vol. 1, n 7, p. 257-260.

Mandelstamm, Valentin (1937), «ll film italiano può andare in America», Cinema, vol. 2, n² 29, p. 157 165.

Mauro, Augusto (1937), «Ma davvero il film italiano può andare in America?», Cinema, vol. 2, n’ 36, p. 420.

Menarini, Alberto (1942), «Autarchia della lingua e terminologia cinematografica», Bianco e nero, vol. $6, n^{\circ} 2$, p. 41-54.

Migliorini, Bruno (1941), «Per una terminologia cinematografica italiana», Bianco e nero, vol. 5, n’ 5, p. 22-29.

Milano, Paolo (1938), «L'italiano del cinema», Cinema, vol. 3, n 49, p. 10-11.

Montesanti, Fausto (1941a), «Della ispirazione cinematografica», Cinema», vol. 6, n’ 109, p. 280-281.

Montesanti, Fausto (1941b), «Replica», Cinema, vol. 6, n¹31, p. 364.

Pac. [forse Giulio Pacuvio?] (1938a), «Appunti», Scenario, vol. 7, n² 2, p. 68.

Pac. [forse Giulio Pacuvio?] (1938b), «Recitare al cinema», Scenario, vol. 7, n³ 3, p. 117.

Palmieri, E. Ferdinando (1943), «Questa critica», L'illustrazione italiana, vol. 70, n’ 49, p. 913.

Palmieri, E. Ferdinando (1944), «Questo pubblico», L'illustrazione italiana, vol. 71, n 9, p. 160.

Pannunzio, Mario (1938), «Recitare al cinema», Scenario, vol. 7, n² 2, p. 65-67.

Paolella, Roberto (1943), «Origine e senso dell'espressione cinematografica. Dal muto al parlato», Cinema, vol. 8, n¹61, p. 140-142.

Patuelli, Raffaello (1936), «ll "Dipartimento dell'educazione" ovvero: Il gergo dei film tradotti», Lo schermo, vol. 2, n`5, p. 28-31.

Pavolini, Corrado (1936), «Tradurre un film», Cinema, vol. 1, n 5, p. 180-181.

Il Pignolo di turno (1940), «La caccia agli errori», Cinema, vol. 5, n 93, p. 326. 
Pirandello, Luigi (1929), «Se il film parlante abolirà il teatro», Corriere della Sera, 16 giugno, p. 3.

Pirandello, Luigi (1939), «Dramma e sonoro» [1929], Cinema, vol. 4, n’ 81, p. 277-278.

Pirandello, Luigi (1940), «Dialettalità» [1921], Scenario, vol. 9, n 1, p. 17.

Ramperti, Marco (1942), «In viaggio per una grammatica», L'illustrazione italiana, vol. 69, n’35, p. 236.

Ramperti, Marco (1943), «La rivincita di Goldoni. Il film della "Locandiera"», L'illustrazione italiana, vol. $70, n^{\circ} 25$, p. 532.

Rossi, Giuseppe (1940), «Cronache della produzione italiana», Lo schermo, vol. 6, n 11, p. 9-17.

Rossi, Vittorio G. (1938), «Tre osservazioni», Cinema, vol. 3, n5 52, p. 112-113.

S. [forse G.V. Sampieri?] (1938), «Volto ed anima italiani per i film italiani», Lo schermo, vol. 4, n’2, p. 21-22.

S.a. (1936), «Gli inconvenienti del parlato», Lo schermo, vol. 2, n 4, p. 33.

S.a. (1940), «Dialetto e linguaggio teatrale», Scenario, vol. 9, n 1, p. 16.

S.a. (1944), «Primizie dello schermo. Ciò che si prepara nei cantieri del doppiaggio», L'illustrazione italiana, vol. 71, n' 35, p. 537.

Salvioni, Emilia (1937), «Una piaga: il cartonaggio», Lo schermo, vol. 3, nº 6, p. 31-32.

Sanminiatelli, Bino (1939), «Libri e pellicola», Cinema, vol. 4, n 78, p. 191-194.

Spagnol, Tito A. (1938), «Le parole per le immagini», Cinema, vol. 3, n 42, p. 188-189.

Spaini, Alberto (1936), «ll cinema è giunto a Shakespeare», Scenario, vol. 5, n 1, p. 10-13.

Spaini, Alberto (1942), «Dalla scena allo schermo», Scenario, vol. 11, n’ 9, p. 309-311.

Uccello, Paolo (1937), «La tecnica e l'arte del doppiato», Bianco e nero, vol. 1, n’ 5, p. 40-55.

Uccello, Paolo (1939), «Come si parla al microfono», Bianco e nero, vol. 3, n’ 10, p. 81-91.

Uccello, Paolo (1942), «ll problema estetico del suono nel film», Lo schermo, vol. 8, n’ 4, p. 29-30.

Usigli, Arrigo [e redazione] (1939), «Difetti e rimedi. Replica sul "sonoro”», Cinema, vol. 4, n 63, p. 88.

Vecchietti, Giorgio [sotto lo pseudonimo di L'uomo ombra] (1936), «Preferisco i fagioli», Lo schermo, vol. 2, n' 10, p. 23-24.

Vecchietti, Giorgio (1938), «ll documentario fa da sé», Cinema, vol. 3, n 41, p. 163-164. 


\section{Fonti secondarie}

BIZ = Stoppelli, Pasquale (ed.), Biblioteca Italiana Zanichelli. DVD-ROM per Windows per la ricerca in testi, biografie, trame e concordanze della Letteratura italiana, Bologna, Zanichelli, 2010.

Bonghi, Ruggero (1856/1884), Perché la letteratura italiana non sia popolare in Italia, Milano, Perelli; IV ed., Napoli, Morano, 1884, da cui si cita.

Brunetta, Gian Piero (2001), Storia del cinema italiano, 4 voll., Roma, Editori Runiti (II ed.).

Caldiron, Orio (2002), «Introduzione», in Caldiron, Orio (ed.), Cinema 1936- 1943. Prima del Neorealismo, Roma, Fondazione Scuola Nazionale di Cinema, p. 9-14.

De Gaetano, Roberto (2014), «Introduzione. Il cinema senza uniforme», in De Gaetano (2014: 7-39).

De Gaetano, Roberto (ed.) (2014-2015), Lessico del cinema italiano, 3 voll., Mimesis, Sesto San Giovanni.

Demartini, Silvia (2014), Grammatica e grammatiche in Italia nella prima metà del Novecento. Il dibattito linguistico e la produzione testuale, Firenze, Cesati.

Fornaciari, Raffaello (1881), Sintassi italiana dell'uso moderno, Firenze, Sansoni.

Giovanardi, Claudio e Pietro Trifone (2015), La lingua del teatro, Bologna, il Mulino.

Gramsci, Antonio (1996), Letteratura e vita nazionale, ed. Valentino Gerretana, Roma, Editori Riuniti (III ed.; I ed. 1975).

Massara, Giuseppe (ed.) (2007), La lingua invisibile. Aspetti teorici e tecnici del doppiaggio in Italia, Roma, Nuova Editrice Universitaria.

Palermo, Massimo (2006), «ll tredicesimo pronome atono», Studi linguistici italiani, vol. 32, p. 109-122.

Pasolini, Pier Paolo (1991), Empirismo eretico, Garzanti, Milano (I ed. 1972).

Pavesi, Maria (2005), La traduzione filmica, Roma, Carocci.

Perego, Elisa e Christopher Taylor (ed.) (2012), Tradurre l'audiovisivo, Roma, Carocci.

Raffaelli, Sergio (1992), La lingua filmata. Didascalie e dialoghi nel cinema italiano, Firenze, Le Lettere.

Raffaelli, Sergio (1993), «Un "Lei” politico. Cronaca del bando fascista (gennaio-aprile 1938)», in Omaggio a Gianfranco Folena, Padova, Editoriale Programma, p. 2061-2073.

Raffaelli, Sergio (2000), «Leopardi, Manzoni e Pirandello purgati dalla censura fascista: niente "lei”», in Studi di letteratura, critica e linguistica offerti a Riccardo Scrivano, Roma, Bulzoni, p. 129-145.

Raffaelli, Sergio (2015), Parole di film. Studi cinematografici 1961-2010, ed. Massimo Fanfani, Firenze, Franco Cesati. 
Rossi, Fabio (1999), Le parole dello schermo. Analisi linguistica del parlato di sei film dal 1948 al 1957, Bulzoni, Roma.

Rossi, Fabio (2006), Il linguaggio cinematografico, Aracne, Roma.

Rossi, Fabio (2015a), «Dalla questione della lingua all'aggressione linguistica: le idee sulla lingua nei giornali italiani dell'ultimo decennio», Circula, n’ 1, p. 173-195.

Rossi, Fabio (2015b), «Lingua», in De Gaetano (2015: 141-213).

Rossi, Fabio (a cura di) 2016. La parola e l'immagine agli albori del Neorealismo. Le questioni linguistiche nei periodici di spettacolo in Italia (1936-1945), Firenze, Cesati.

Ruffin, Valentina e Patrizia D’Agostino (1997), Dialoghi di regime. La lingua del cinema degli anni trenta, introduzione di Gian Piero Brunetta, Roma, Bulzoni.

Santulli, Francesca (2015), «La riflessione metalinguistica sulla stampa italiana: oltre l'epicedio?», Circula, n², p. 55-75.

Serianni, Luca (1989), Saggi di storia linguistica italiana, Napoli, Morano.

Trifone, Pietro (2017), Pocoinchiostro. Storia dell'italiano comune, Bologna, il Mulino.

Zavattini, Cesare (1979), Neorealismo ecc.», ed. Mino Argentieri, Milano, Bompiani. 